“C 2019 IEEE. Personal use of this material is permitted. Permission from IEEE must be obtained for all other uses, in any current or future media, including reprinting/republishing this material for advertising or promotional purposes, creating new collective works, for resale or redistribution to servers or lists, or reuse of any copyrighted component of this work in other works." 


\title{
A Semi-supervised Recurrent Convolutional Attention Model for Human Activity Recognition
}

\author{
Kaixuan Chen, Student Member, IEEE, Lina Yao, Member, IEEE, Dalin Zhang, Xianzhi Wang, Member, IEEE, \\ Xiaojun Chang, Member, IEEE, and Feiping Nie, Member, IEEE,
}

\begin{abstract}
Recent years have witnessed the success of deep learning methods in human activity recognition (HAR). The longstanding shortage of labeled activity data inherently calls for a plethora of semi-supervised learning methods, and one of the most challenging and common issues with semi-supervised learning is the imbalanced distribution of labeled data over classes. Although the problem has long existed in broad real-world HAR applications, it is rarely explored in the literature. In this paper, we propose a semi-supervised deep model for imbalanced activity recognition from multimodal wearable sensory data. We aim to address not only the challenges of multimodal sensor data (e.g., inter-person variability and inter-class similarity) but also the limited labeled data and class-imbalance issues simultaneously. In particular, we propose a pattern-balanced semi-supervised framework to extract and preserve diverse latent patterns of activities. Furthermore, we exploit the independence of multimodalities of sensory data and attentively identify salient regions that are indicative of human activities from inputs by our recurrent convolutional attention networks. Our experimental results demonstrate that the proposed model achieves a competitive performance compared to a multitude of state-of-the-art methods, both semi-supervised and supervised ones, with $10 \%$ labeled training data. The results also show the robustness of our method over imbalanced, small training datasets.
\end{abstract}

Index Terms-human activity recognition, semi-supervised learning, class imbalance, attention.

\section{INTRODUCTION}

$\mathbf{H}$ UMAN Activity Recognition (HAR) is a fundamental technique popular in healthcare and surveillance domains [1]. In particular, wearable physical sensor signal processing-based HAR has been widely applied to ubiquitous applications and profoundly revolutionized our daily lives, thanks to its high resistance to environmental variation without significantly violating individual privacy.

Although remarkable efforts have been contributed to different aspects of HAR, three challenges remain for the research community. The first is insufficient labeled observations [2]. Most existing works follow a supervised learning approach [3], [4], thus requiring a significant amount of training data to

K. Chen is with the School of Computer Science and Engineering, UNSW Sydney, NSW 2052, Australia. E-mail: kaixuan.chen@student.unsw.edu.au.

L. Yao is with the School of Computer Science and Engineering, UNSW Sydney, NSW 2052, Australia. E-mail: lina.yao@unsw.edu.au.

D. Zhang is with the School of Computer Science and Engineering, UNSW Sydney, NSW 2052, Australia. E-mail: dalin.zhang@student.unsw.edu.au.

X. Wang is with University of Technology Sydney, NSW 2007, Australia. E-mail: xianzhi.wang@uts.edu.au.

$\mathrm{X}$. Chang is with Monash University, Melbourne, Australia. E-mail: cxj273@gmail.com

F. Nie is with Northwestern Polytechnical University, Xi'an, China. E-mail: feipingnie@gmail.com recognize meaningful activities. However, ground truth annotation is usually both costly and error-prone. Semi-supervised methods, in contrast, additionally leverage unlabeled data to train the model and therefore are considered more promising in many scenarios. Although researchers have already investigated several semi-supervised techniques [5], [6], they neglect the benefit of $\mathrm{c}$ ombining $\mathrm{m}$ ultimodality s ensor data and overlook the inner patterns of each activity. Considering its superiority in dealing with multimodalities, we resort to a co-training method [7]. Previous studies [8] suggest that cotraining algorithms can work well when the multiple views (or multimodalities) are not strongly correlated [9], with each view containing sufficient information to learn a weakly useful classifier a nd $o$ ther $v$ iews $r$ edundant $f$ or $t$ his $v$ iew [ 7]. The above property makes co-training appropriate for the multimodal activity recognition problem.

The second challenge concerns the expense and convenience of labeling activity data, where the class imbalance is often a concurrent issue. Especially in HAR tasks, some activity data (e.g., those related to falls of elder people) are difficult to obtain and label. In fact, semi-supervised learning on imbalanced classification i s e ven $\mathrm{m}$ ore $\mathrm{c}$ hallenging. While most classifiers $t$ end $t \mathrm{o} p$ redict $\mathrm{m}$ ajority $\mathrm{c}$ lass $\mathrm{s}$ amples with high accuracy and treat the minority classes as outliers [10], the situation becomes more severe when only a small amount of data is available. Previous works directly apply undersampling or over-sampling [11], but they are unsuitable for our case as they both change the distribution of the training data. Since in our case, the same individual may perform the same activity in different ways because of stress, fatigue, emotion and other environmental factors, it is reasonable to assume that samples in each class can form several latent patterns. Therefore, we select training samples in each training round in line with the extracted latent patterns to sustain the diversity of activity patterns. Such a pattern-preserving framework maintains the distribution of training data and improves the labeling performance during training.

The third challenge contains two parts and is longstanding for HAR: inter-person variability and inter-class similarity [2]. The inter-person variability means the same activity can be performed differently by different people and inter-class similarity results from the similarity in the behavior patterns of different activities like walking and running. Since deep learning based methods have the strength of modeling the high-level representations of data and have achieved outstanding performance in the field of HAR, we aim to explore more potentials of deep learning models in the field of HAR. We 
train "attentive" deep models to extract the salient information indicative of the true activity to obtain useful information from limited training data and address the influences of the interperson variability and inter-class similarity.

In brief, we deploy a pattern-balanced recurrent convolutional attention model to address the above concerns. Our approach achieves high accuracy on a small size, imbalanced data. To the best of our knowledge, this paper is the first that uses semi-supervised learning in imbalanced activity recognition. The key contributions of this research are as follows:

- We propose a novel method that employs semi-supervised learning for imbalanced HAR, which is a significant challenge yet rarely explored in the literature.

- Considering the influence of class imbalance on limited training data, we propose a pattern-balanced co-training for extracting and preserving the latent activity patterns from imbalanced datasets. The patterns maintain the distribution of training data and improve the robustness of co-training on imbalanced data.

- To better utilize the limited labeled data and get higher labeling accuracy during training, we employ Recurrent Attention Models (RAMs) [12] and let them collaborate to exploit unlabeled samples.

- We compare our model with state-of-the-art methods on three public benchmarked datasets and a new dataset collected in the real world. The experimental results show that our approach achieves competitive performance compared to state-of-the-art semi-supervised and even supervised methods with $10 \%$ labeled training data.

\section{RELATED WORK}

\section{A. Semi-Supervised Learning for Imbalanced HAR}

While deep learning methods achieve high recognition performance in HAR [4], they require a substantial amount of labeled activity data for training. Semi-supervised learning allows leveraging both labeled and unlabeled data to train a recognition system. Some works use self-learning based approaches [6], and some utilize graph-based approached [5], [13]. Nevertheless, these approaches all rely on ad-hoc handcrafted features, which makes it hard and expensive to build a recognition system. [14], [15] resort to deep generative models such as Restricted Boltzmann Machines (RBM) and autoencoders to train the model with a significant amount of unlabeled data and get a well-trained feature extractor. The feature extracted can be further recognized with classifiers trained with labeled data. Methods like these, however, suffer from three defects. Firstly, none of these methods takes advantage of the relations between multimodalities of activity data which is of great significance since modalities carry information from different perspectives and complement each other. Secondly, they fail to explore the potential activity category information of those unlabeled data [4]. The reason why discriminative models cannot directly participate in semi-supervised learning is that the small size of labeled data is not enough for training. In contrast, our method utilizes the disagreement between modalities and introduce attention mechanisms, so deep learners can learn to exploit potential class information of unlabeled samples with small labeled sets.

However, none of these semi-supervised methods consider a more common case where the class distribution is imbalanced. Although some approaches [16], [10] have been proposed to solve the imbalance issue, their performance deteriorates owing to the small data size in semi-supervised learning. Works like [11], [17] are devoted to semi-supervised learning for imbalanced learning, yet they aim at solving a binary classification problem, which is more straightforward than recognizing multiple activities in HAR. So far, semi-supervised learning for imbalanced HAR problems still has not been carefully studied in the literature.

\section{B. Attention Mechanisms}

Attention originates from biology and psychology that implies focusing the power of noticing or thinking on something special to achieve better cognitive processes. Tracing back the history of selecting effective regions using attention mechanisms or similar theories, attention-based RNN model has achieved success in both speech recognition [18] and computer vision [19], [12]. Bahdanau et al. [18] build a vocabulary continuous speech recognition system using an attention-based RNN as it requires fewer training stages, fewer auxiliary data, and less domain expertise. Some works [19], [12] formulate the selection process into a sequential decision task. Our previous work [20] adopts the attention mechanism for HAR. We fuse attention with CNN and RNN to automatically extract the most salient modality-specific features and further convert the information to higher-level representation. In this work, our approach allows the attention mechanism to fully leverage its strengths to strive for a balance from less labeled data.

\section{The Methodology}

\section{A. Overview}

In this section, we propose an integrated system for semi-supervised and imbalanced HAR. Firstly, we propose a pattern-balanced framework that preserves and balances diverse intent patterns of activities. The proposed framework improves the performance of conventional co-training under imbalanced labeled data (Section III-B). Secondly, considering the limited labeled data, we aim at maximizing the utilization of salient features and ignoring the irrelevant signals. We introduce attention mechanism and deploy recurrent convolutional attention models to get better labeling accuracy on limited labeled data and deal with the inter-person variability and inter-class similarity of HAR (Section III-C).

Since the modalities in our case satisfy the sufficiency, redundancy and weak relations that co-training requires, we develop an effective semi-supervised framework based on cotraining method [7] to handle limited labeled data.

The basic framework of co-training is as follows: 1) The training data contains two parts: labeled set $\mathcal{L}$ and unlabeled set $\mathcal{U}$. 2) Three classifiers are trained on $\mathcal{L}$ of acceleration, angular velocity, and magnetism, respectively. 3) Each model is applied to $\mathcal{U}$ to make a prediction and vote to label the most confident samples. These selected samples are removed from 


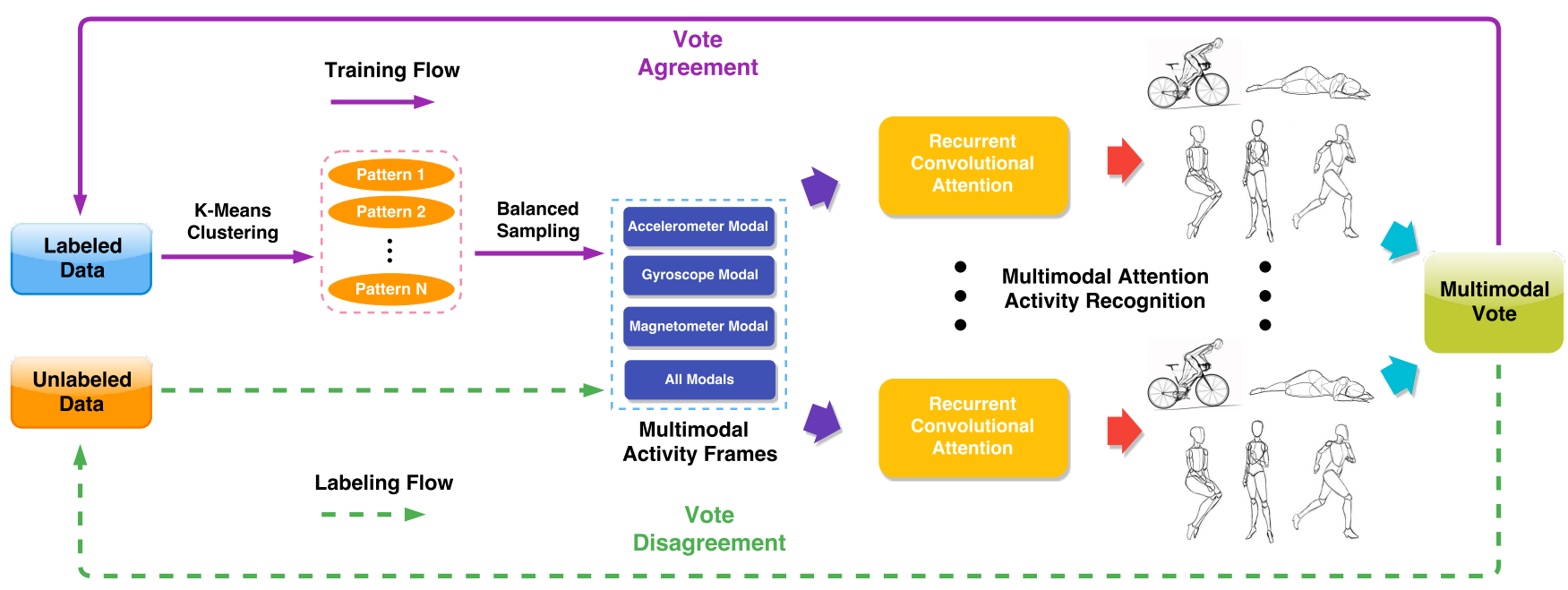

Fig. 1: Workflow of the proposed pattern-balanced co-training framework. The framework contains two flows. 1) Training flow (indicated as solid lines): Labeled data are categorized into $N$ patterns via k-means clustering and data of patterns are sampled evenly to train multimodal classifiers. 2) Labeling flow (indicated as dashed lines): Predict the unlabeled data with trained models. If most of the classifiers reach an agreement on predicting a sample, this sample is labeled, otherwise, keep it unlabeled.

$\mathcal{U}$ and added to $\mathcal{L}$ to improve the classifiers in the following training rounds. 4) Repeat steps 2) - 3) until no more samples can be voted or $\mathcal{U}$ turns empty. 5) Train a classifier with the final $\mathcal{L}$ and all modalities. Owing to the prevalence and excellent performance of deep learning methods in HAR, we deploy deep learning classifiers and embed them into the cotraining framework.

While the framework above seems to be valid, we observe that the labeling accuracy decreases with training rounds since the problem of class imbalance is severe when the labeled data size is small. The practical difficulty of obtaining and labeling some specific activity data (e.g., falls of elder people) make the problem even more challenging. Therefore, we propose to tackle the class imbalance by pattern-balanced training in Section III-B.

\section{B. Class Imbalance Mitigation}

Our proposed pattern-balanced training is robust to classimbalanced labeled data. Fig. 1 shows the overall workflow of our framework. The workflow contains a training flow and a labeling flow.

Training Flow. Its goal is to train weak classifiers with labeled data so that they can vote to label samples from unlabeled data with high accuracy even in class-imbalanced situations. For clarity, the labeled set $\mathcal{L}$ contains $L$ labeled samples. Each sample $(\mathbf{x}, y)$ consists of a vector that represents the collected sensory data $\mathbf{x}$ and the activity label $y$. Since most IMUs used in HAR community contains three inertial sensors, an accelerometer, a gyroscope and a magnetometer, we suppose that

$$
\mathbf{x}=(\text { Acc }, \text { Gyro, } \text { Magn })
$$

and

$$
y \in[1 \ldots C]
$$

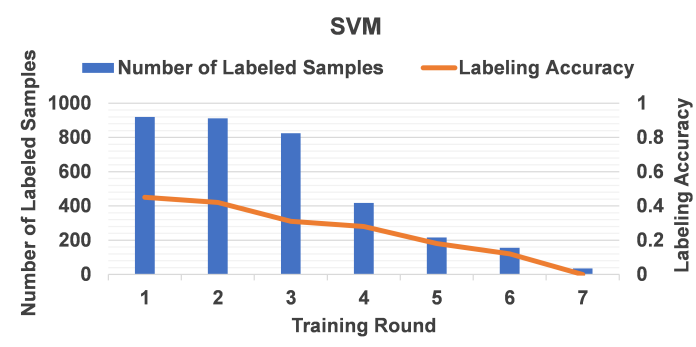

(a) SVM

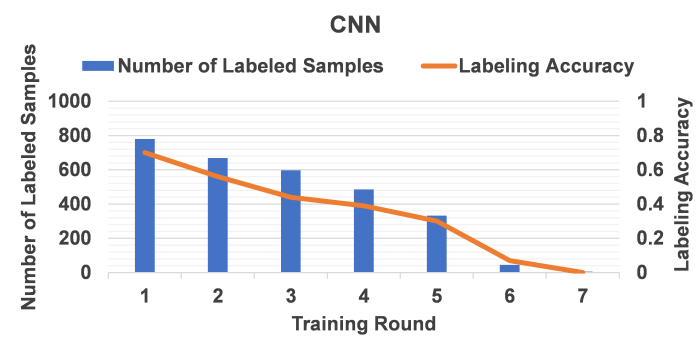

(b) $\mathrm{CNN}$

Fig. 2: Labeling Accuracy and Numbers of Labeled Samples vs. Training Rounds on 2000 original labeled data and 18000 unlabeled data. The orange lines represent the labeling accuracy and the blue bars represent the numbers of samples labeled in each training round.

where $C$ denotes the number of activity classes. To focus on the class imbalance problem, $\mathcal{L}$ is separated to $C$ classes according to their labels so that

$$
\mathcal{L}=\bigcup_{i=0}^{C} \mathcal{C}_{i}
$$


where

$$
\mathcal{C}_{i}=\{(\mathbf{x}, y) \mid y=i\}=\left\{\left(\mathbf{x}_{0}, i\right), \ldots\left(\mathbf{x}_{j}, i\right) \ldots\left(\mathbf{x}_{\operatorname{Card}\left(\mathcal{C}_{i}\right)}, i\right)\right\}
$$

and $\operatorname{Card}\left(\mathcal{C}_{i}\right)$ represents the cardinality of $\mathcal{C}_{i}$.

A basic solution to class imbalance is to over-sample smallclass data or under-sample large-class data [21], but it may change the distribution of training data and lead to "covariate shift". On the other hand, considering the intra-class variation of HAR, we aim at preserving the diversity of patterns within each class to avoid the distribution shift. As the latent patterns rely on expertise, we adopt k-means clustering to each class $\mathcal{C}_{i}$ to extract activity patterns by minimizing the measurement:

$$
D=\sum_{m=1}^{\operatorname{Card}\left(\mathcal{C}_{i}\right)} \sum_{k=1}^{K_{i}} \boldsymbol{\mu}_{k m}\left\|\mathbf{x}_{m}-\mathbf{z}_{k}\right\|
$$

where $K_{i}$ denotes the number of clusters of $\mathcal{C}_{i}$ and it is adaptively decided by its covariance [22]. $\boldsymbol{\mu}_{k m}=1$ if $\mathbf{x}_{m}$ belongs to the cluster $\mathcal{Z}_{k}$ with center $\mathbf{z}_{k}$; otherwise, $\boldsymbol{\mu}_{k m}=0$. Therefore,

$$
\mathcal{C}_{i}=\bigcup_{n=0}^{K_{i}} \mathcal{Z}_{n}
$$

After extracting the activity patterns $\mathcal{Z}$, we apply oversampling and under-sampling to classes to maintain the distribution. In particular, we randomly select a same number of samples from patterns $\mathcal{Z}$ of classes $\mathcal{C}$ to make sure that samples from all patterns evenly participate in the next training round. We conduct clustering and sampling in the training flow rather than the labeling flow to avoid repeatedly labeling samples. Then, four classifiers are trained separately for acceleration, angular velocity, magnetism and the combination of all modalities. All modalities are treated as the fourth modality to guarantee the labeling accuracy.

Labeling Flow. In the labeling flow, the trained classifiers are applied to unlabeled data to make a prediction. Four classifiers vote to select confident samples and label them. These selected samples are removed from the unlabeled set $\mathcal{U}$ and added to $\mathcal{L}$. Next, the training flow and the labeling flow are repeated until no confident samples can be labeled or $\mathcal{U}$ is empty. The last step is to train a classifier on the final labeled set with all modalities. In our framework, we only fine-tune the fourth classifier (which is repeatedly trained in the training flow) with the newly-labeled samples. The target of the labeling flow is to leverage the sufficiency and redundancy of multi-modalities so that four classifiers can learn from each other.

\section{Limited Data Exploitation}

Even with pattern-balanced training, another issue hinders the HAR concerning imbalanced, small labeled sets. After the sampling, the already limited labeled set becomes even smaller. As a result, it is hard to train satisfactory models with so limited labeled data in our case, especially when HAR data naturally suffers from inter-person variability and interclass similarity. Fig. 2 shows the labeling accuracy and the numbers of samples labeled in each training round with 2000 balanced labeled data and 18000 unlabeled data. Firstly, CNN enjoys the merit of deep learning, so the labeling accuracy of $\mathrm{CNN}$ is higher than that of SVM in the first round. However, as the models only have 2000 original labeled samples, the labeling accuracy is low ( 0.45 for SVM and 0.72 for CNN). As SVM labeled about 900 samples with 0.45 accuracy, which means they introduce 550 falsely labeled samples to $\mathcal{L}$ approximately. The falsely labeled samples create a vicious circle and further decreases the labeling accuracy in the following training rounds until the accuracy is 0 . With respect to $\mathrm{CNN}$, as $\mathrm{CNN}$ has a relatively higher accuracy in the first round, it has a more stable decrease in both the labeled data number and the labeling accuracy, but still cannot avoid the continuously decreasing accuracy. Therefore, it is necessary to train classifiers that can fully exploit salient features from limited data and achieve high labeling accuracy. In this section, we use RAMs [12] which extracts the informative features. They can learn modality-specific information and distinguish disagreement among the modalities so that these models can learn from each other and do not incorrectly vote samples to the same labels.

Motivations. Intuitively, the motion of different body parts has various contributions to different activities [23]. For example, jumping involves legs while running is related to both arms and legs; another example is that recognizing patterns of walking depends more on the acceleration of legs while distinguishing sitting from lying would rely more on the orientation of sensor placement. With these characteristics of motion data considered, the natural idea is to focus on the most highly contributing part of several modality data. Inspired by the procedures of human brains processing visual information, we introduce the attention mechanism into HAR systems. RAM is particularly efficient in the scenario when the number of labeled data is limited as it maximizes the effect of useful information and alleviates the influence of inter-person variability and inter-class similarity. Hence, it can work effectively even when the labeled data size is small.

Fig. 3 shows the basic structure, where the whole process sits on a core LSTM. At each time step $t$, the model only focuses on a small patch which is called a glimpse. To extract the most salient patch, we train the model using reinforcement learning [12]. RAM consists of four key components: (i) a glimpse layer, (ii) a convolutional network, (iii) a glimpse representation layer, and (iv) a core recurrent attention unit. We explain the details in the following.

Glimpse Layer. The first part of the proposed model is a glimpse layer. The glimpse layer not only avoids the system processing the whole data in their entirety but also maximally eliminates the information loss raised by traditional dimensionality reduction and feature selection [12], [19]. As sensory data do not have fixed ordering arrangement, we preprocess the multimodal sensor data by transforming them from sequences to matrices with the arrangement algorithm proposed in our previous work [20]. This arrangement extracts the full correlations between feature pairs so that the glimpses selected may contain relations between both adjacent and nonadjacent features. Inspired by the human visual system, in RAM, each input matrix $I$ will be "understood" within $T$ glimpses. Simulating the process of how the human eye works, 


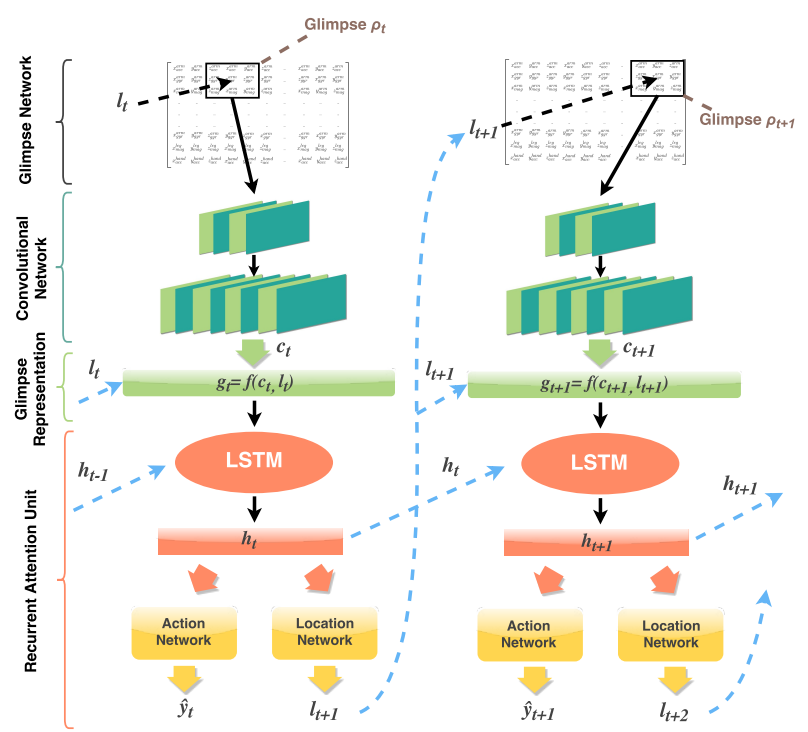

Fig. 3: RAM. The input data are represented as matrices. At each time step $t$, a small glimpse patch is extracted with the glimpse layer and processed by a convolutional network. The processed glimpse information is then encoded with location information in the following step. A recurrent network with two sub-networks predict the activity at the current time step and decide the glimpse location for the next time step.

RAM extracts a glimpse region denoted by $\rho_{t}$ from the input matrix $I$ at the location $l_{t}$ at each time step $t$.

Convolutional Network. Human visual system converts retina images into brain signals via the optic nerves. Likewise, we convert the glimpse directly extracted from the input matrix into higher-level information. RAM uses a convolutional network to encode $\rho_{t}$ to be $c_{t}$, parameterized by $\theta_{c}$, which generates a high-level representation that characterizes the local salience of the low-level sensor data:

$$
c_{t}=\operatorname{Conv}\left(\rho_{t} ; \theta_{c}\right)
$$

Glimpse Representation Layer. The glimpse needs to be further processed by a glimpse representation layer [12], [19]. $c_{t}$ and the location $l_{t}$ are linearly transformed independently with two linear layers parameterized by $\theta_{g}^{c}$ and $\theta_{g}^{l}$, respectively. Next, the summation of these two parts is further transformed with another linear layer parameterized by $\theta_{g}$ and a rectified linear unit. The whole process is summarized as follows:

$$
g_{t}=f_{g}\left(c_{t}, l_{t} ; \theta_{g}^{c}, \theta_{g}^{l}, \theta_{g}\right)=\operatorname{relu}\left(L\left(L\left(c_{t}\right)+L\left(l_{t}\right)\right)\right)
$$

where $L(\bullet)$ denotes a linear transformation. Therefore, the glimpse representation $g_{t}$ finally contains information from both "what" $\left(c_{t}\right)$ and "where" $\left(l_{t}\right)$.

Recurrent Attention Unit. We use a recurrent neural network as the core to process data step by step within several glimpses. As Fig. 3 shows, the basic structure of the recurrent attention unit is an LSTM. At each time step $t$, the LSTM receives the glimpse $g_{t}$ and the previous hidden state $h_{t-1}$ as the inputs parameterized by $\theta_{h}$. Meanwhile, it outputs the current hidden state $h_{t}$ according to the equation:

$$
h_{t}=f_{h}\left(h_{t-1}, g_{t} ; \theta_{h}\right)
$$

The recurrent attention unit also contains two sub-networks: the location network and the action network. These two subnetworks receive the hidden state $h_{t}$ as the input to decide the next glimpse location $l_{t+1}$ and the current action $a_{t}$. The location network outputs the location at time $t+1$ stochastically according to the location policy defined by a Gaussian distribution stochastic process, parameterized by the location network $f_{l}\left(h_{t} ; \theta_{l}\right)$ :

$$
l_{t+1} \sim P\left(\cdot \mid f_{l}\left(h_{t} ; \theta_{l}\right)\right)
$$

Similarly, the action network outputs the corresponding action at time $t$ and predicts the activity label $\hat{y}_{t}$ given the hidden state $h_{t}$. The action $\hat{y}_{T}$ at the last time step $T$ indicates the final prediction of the activity. $\hat{y}_{t}$ obeys the distribution parameterized by $f\left(h_{t} ; \theta_{a}\right)$. Owing to its prediction function, the network uses a softmax formulation:

$$
a_{t}=\hat{y}_{t}=f_{a}\left(h_{t} ; \theta_{a}\right)=\operatorname{softmax}\left(L\left(h_{t}\right)\right)
$$

Training and Optimization. Our proposed model involves the parameters of the convolutional network, the glimpse representation layer and the two sub-networks, $\Theta=$ $\theta_{c}, \theta_{g}^{c}, \theta_{g}^{l}, \theta_{g}, \theta_{h}, \theta_{a}, \theta_{l}$. Since the action network relies on classification methods, $\theta_{a}$ can be trained by optimizing the cross-entropy loss and the backpropagation. However, we expect the location network to be able to select a sequence of salient regions from input matrices adaptively. In view that the location network is stochastic and non-differentiable, the salient region selection problem can also be seen as a control problem, and it can be trained by reinforcement methods to learn the optimal policies. Based on the above discussion, we deploy a Partially Observable Markov Decision Process (POMDP) to solve the training and optimization problems [24]. In particular, we call the sequence of the input, location and action pairs, $s_{1: t}=\mathbf{x}, l_{1}, \hat{y}_{1} ; \ldots \mathbf{x}, l_{t}, \hat{y}_{t}$, an attention sequence and use this sequence to represent the order of the regions that the model focused on. In our case, the location network is formulated as a random stochastic process (the Gaussian distribution) parameterized by $\Theta$. Each time after the location selection, the prediction $\hat{y}$ is evaluated to back feed a reward $r$ for conducting the backpropagation training process. $r_{t}=1$ if $\hat{y}_{t}=y_{t}$ and 0 otherwise. The process is also defined as policy gradient. Our goal is to maximize the simulated rewards $R$ using gradient. Given a sample $x$ with reward $f(x)$ and probability $p(x)$, the gradient can be 
calculated as follows:

$$
\begin{aligned}
\nabla_{\theta} E_{x}[f(x)] & =\nabla_{\theta} \sum_{x} p(x) f(x) \\
& =\sum_{x} \nabla_{\theta} p(x) f(x) \\
& =\sum_{x} p(x) \frac{\nabla_{\theta} p(x)}{p(x)} f(x) \\
& =\sum_{x} p(x)\left(\nabla_{\theta} \log p(x)\right) f(x) \\
& =E_{x}\left[f(x)\left(\nabla_{\theta} \log p(x)\right)\right]
\end{aligned}
$$

In our case, given the reward $R$ and the attention sequence $s_{1: T}$, the reward function to be maximized is as follows:

$$
J(\Theta)=\mathbb{E}_{p\left(s_{1: T} ; \Theta\right)}\left[\sum_{t=1}^{T} r_{t}\right]=\mathbb{E}_{p\left(s_{1: T} ; \Theta\right)}[R]
$$

By considering the training problem as a POMDP, a sample approximation to the gradient is calculated as follows according to the REINFORCE rule [25]:

$$
\nabla_{\Theta} J=\sum_{t=1}^{T} \mathbb{E}_{p\left(s_{1: t} ; \Theta\right)}\left[R \nabla_{\Theta} \log \pi\left(y \mid s_{1: t} ; \Theta\right)\right]
$$

We use Monte Carlo sampling which utilizes randomness to yield results that might be deterministic theoretically. Supposing $M$ is the number of Monte Carlo sampling copies, we duplicate the same input for $M$ times and average them as the prediction results to overcome the randomness in the network, where the $M$ duplication generates $M$ subtly different results owing to the stochasticity, we have:

$$
\nabla_{\Theta} J \approx \frac{1}{M} \sum_{i=1}^{M} R^{(i)} \sum_{t=1}^{T} \nabla_{\Theta} \log \pi\left(y^{(i)} \mid s_{1: t}^{i} ; \Theta\right)
$$

where $i$ denotes the $i^{t h}$ training sample, $y^{(i)}$ is the correct label for the $i^{t h}$ sample, $\nabla_{\Theta} \log \pi\left(y^{(i)} \mid s_{1: t}^{i} ; \Theta\right)$ is the gradient of LSTM calculated by backpropagation, and $M$ denotes the number of Monte Carlo sampling copies used for overcoming the randomness of the networks.

Therefore, although the best attention sequences are unknown, RAM can learn the optimal policy in the light of the reward. The experiments show that RAM outperforms the state-of-the-art in the initial phase of co-training.

\section{EXPERIMENTS}

In this section, we evaluate the proposed method on four datasets. Three of them, MHEALTH [29], PAMAP2 [30] and UCI HAR [1], are public benchmarked datasets on activity recognition. They are the latest available multimodal wearable sensor-based datasets with complete annotation. The other one, MARS (Multimodal Activity Recognition with Sensing), is a real-world dataset that we collected to re-examine the practicability of the proposed method. This dataset is collected while 8 participants (six males, two females) are performing five basic activities (sitting, standing, walking, ascending stairs and descending stairs).
We firstly compare our method with different state-of-theart works and baselines under both supervised and semisupervised schemes. Then we explore the robustness to class imbalance by comparing the proposed method with stateof-the-art and baselines in five class imbalance situations. Thirdly, we perform a detailed ablation study to examine the contributions of the proposed components to the prediction performance. Lastly, we visualized the selected features by the attention model. Due to the page constraint, some details and experimental results are presented in the supplementary materials. The materials contain model implementation, confusion matrices, time latency comparison, empirical studies on annotation scarcity and class imbalance, analysis of training evolution and hyper-parameter study.

\section{A. Robustness to Annotation Scarcity}

To verify our semi-supervised approach's robustness to data scarcity, we extensively compare our model with a set of state-of-the-art and baseline methods trained with different numbers of labeled samples. The compared methods include both supervised approaches and semi-supervised approaches. We compare our approach with supervised approaches not only to exhibit the robustness to annotation scarcity but also to show that our classifiers are stronger than these supervised methods.

Table I presents the comparison between the proposed approach and the state-of-the-art methods as well as baselines. The datasets used in these experiments are class balanced. The notation "sup" indicates supervised methods while "semisup" indicates semi-supervised methods. For fairness, we only deploy the supervised methods with sufficient labeled data. Similar to [3], the experiments conducted on the PAMAP2 and MHEALTH datasets perform background activity recognition tasks [30]. The activities belong to six classes: lying, sitting/standing, walking, running, cycling and other activities. All the models are implemented on the above four datasets with parameters either indicated in the literature or via careful parameter tuning. We can observe that from 1,000 labeled data to 2,000 labeled data, there is a relatively large gap. However, the performance of the model trained with 2,000 labeled samples is only slightly worse than that of the model trained with 20,000 labeled samples. Moreover, with only 2,000 labeled samples and 18,000 unlabeled samples, our approach achieves competitive or better results than the supervised methods. Even with only 1,000 samples, the results are acceptable. Compared to the other semi-supervised methods, the proposed method shows significant improvement (at least 9\%), which demonstrates the effectiveness of the patternbalanced co-training framework. Also, when we train RAM in a supervised manner (i.e., with 20,000 labeled samples), the results are better than the state-of-the-art supervised methods, indicating that RAM outperforms the other methods.

\section{B. Robustness to Class Imbalance}

We conduct experiments in five class imbalance situations to explore the robustness to class imbalance. As the background activity class "others" makes a considerable impact 
TABLE I: The classification accuracy of proposed approach and six state-of-the-art methods and baseline methods on different sizes of labeled sets. The numbers of the labeled data used by the semi-supervised models are denoted in each column (x) and the numbers of the unlabeled samples are 20,000-x. * indicates our approach with 2,000 labeled data outperforms or is competitive with the compared methods with full labeled data.

\begin{tabular}{|c|c|c|c|c|c|c|c|c|c|c|}
\hline \multirow{2}{*}{ Dataset } & \multirow{2}{*}{ Method } & \multirow{2}{*}{ Training Scheme } & \multicolumn{8}{|c|}{ Labeled Samples } \\
\hline & & & 1000 & 2000 & 5000 & 7000 & 10000 & 13000 & 17000 & 20000 \\
\hline \multirow{8}{*}{ MHEALTH } & Multichannel CNN [4] & Sup & 0.6226 & 0.6285 & 0.6936 & 0.7108 & 0.7573 & 0.8304 & 0.8587 & 0.8719 \\
\hline & Attention [20] & Sup & 0.8152 & 0.8573 & 0.8970 & 0.9002 & 0.9178 & 0.9149 & 0.9242 & 0.9392 \\
\hline & Modality-Specific [26] & Sup & 0.6358 & 0.6655 & 0.7616 & 0.7697 & 0.8336 & 0.8486 & 0.8620 & 0.8967 \\
\hline & Co-Training+CNN & Semi-Sup & 0.6331 & 0.6495 & 0.6538 & 0.7029 & 0.7932 & 0.8110 & 0.8503 & 0.8604 \\
\hline & Diversity Preserving [27] & Semi-Sup & 0.7023 & 0.7572 & 0.8271 & 0.8535 & 0.8721 & 0.8789 & 0.8892 & 0.8904 \\
\hline & Tri-Net [28] & Semi-Sup & 0.6938 & 0.6963 & 0.7288 & 0.8076 & 0.8582 & 0.8603 & 0.8661 & 0.8754 \\
\hline & Our Approach & Sup & 0.8059 & 0.8327 & 0.8714 & 0.8924 & 0.8935 & 0.9194 & 0.9326 & 0.9405 \\
\hline & Our Approach & Semi-Sup & 0.8895 & 0.9194 & 0.9208 & 0.9264 & 0.9385 & 0.9425 & 0.9411 & 0.9405 \\
\hline \multirow{8}{*}{ PAMAP2 } & Multichannel CNN [4] & Sup & 0.5405 & 0.6255 & 0.6435 & 0.6483 & 0.7167 & 0.7400 & 0.7976 & 0.8116 \\
\hline & Attention [20] & Sup & 0.6443 & 0.7484 & 0.7756 & 0.7785 & 0.7869 & 0.8045 & 0.8187 & 0.8239 \\
\hline & Modality-Specific [26] & Sup & 0.5578 & 0.5641 & 0.6169 & 0.6949 & 0.7728 & 0.7949 & 0.8040 & 0.8208 \\
\hline & Co-Training+CNN & Semi-Sup & 0.5998 & 0.6042 & 0.6321 & 0.6612 & 0.6857 & 0.7328 & 0.7768 & 0.7922 \\
\hline & Diversity Preserving [27] & Semi-Sup & 0.6412 & 0.6471 & 0.6783 & 0.7394 & 0.7729 & 0.7861 & 0.7913 & 0.8023 \\
\hline & Tri-Net [28] & Semi-Sup & 0.6329 & 0.6429 & 0.6541 & 0.6954 & 0.7252 & 0.7624 & 0.7955 & 0.8088 \\
\hline & Our Approach & Sup & 0.6289 & 0.7305 & 0.7654 & 0.7749 & 0.7935 & 0.8069 & 0.8228 & 0.8342 \\
\hline & Our Approach & Semi-Sup & 0.7338 & 0.8125 & 0.8137 & 0.8135 & 0.8204 & 0.8329 & 0.8318 & 0.8342 \\
\hline \multirow{8}{*}{ UCI HAR } & Multichannel CNN [4] & Sup & 0.5355 & 0.5531 & 0.5584 & 0.5696 & 0.6724 & 0.7368 & 0.7469 & 0.7586 \\
\hline & Attention [20] & Sup & 0.6683 & 0.6969 & 0.6996 & 0.7104 & 0.7297 & 0.7733 & 0.8073 & 0.8129 \\
\hline & Modality-Specific [26] & Sup & 0.5465 & 0.5502 & 0.5879 & 0.6269 & 0.6785 & 0.7360 & 0.7582 & 0.7753 \\
\hline & Co-Training+CNN & Semi-Sup & 0.5212 & 0.5739 & 0.6215 & 0.7106 & 0.7059 & 0.7248 & 0.7201 & 0.7336 \\
\hline & Diversity Preserving [27] & Semi-Sup & 0.6113 & 0.6502 & 0.7054 & 0.7008 & 0.7129 & 0.7310 & 0.7316 & 0.7408 \\
\hline & Tri-Net [28] & Semi-Sup & 0.6156 & 0.6446 & 0.6607 & 0.6675 & 0.6852 & 0.7013 & 0.7207 & 0.7365 \\
\hline & Our Approach & Sup & 0.6427 & 0.6863 & 0.6981 & 0.7124 & 0.7249 & 0.7554 & 0.7804 & 0.8132 \\
\hline & Our Approach & Semi-Sup & 0.7281 & 0.7762 & 0.7818 & 0.7851 & 0.8073 & 0.8143 & 0.8084 & 0.8132 \\
\hline \multirow{8}{*}{ MARS } & Multichannel CNN [4] & Sup & 0.6628 & 0.6699 & 0.6868 & 0.7029 & 0.7351 & 0.7559 & 0.7921 & 0.8138 \\
\hline & Attention [20] & Sup & 0.7223 & 0.7832 & 0.8018 & 0.8357 & 0.8434 & 0.8408 & 0.8497 & 0.8538 \\
\hline & Modality-Specific [26] & Sup & 0.6751 & 0.6787 & 0.6867 & 0.6884 & 0.7009 & 0.7334 & 0.7680 & 0.8354 \\
\hline & Co-Training+CNN & Semi-Sup & 0.6442 & 0.6538 & 0.6968 & 0.7162 & 0.7321 & 0.7259 & 0.7954 & 0.8125 \\
\hline & Diversity Preserving [27] & Semi-Sup & 0.7158 & 0.7370 & 0.8157 & 0.8294 & 0.8238 & 0.8169 & 0.8191 & 0.8208 \\
\hline & Tri-Net [28] & Semi-Sup & 0.6904 & 0.7084 & 0.7376 & 0.7594 & 0.7691 & 0.7857 & 0.8052 & 0.8193 \\
\hline & Our Approach & Sup & 0.7058 & 0.7444 & 0.7689 & 0.7841 & 0.7902 & 0.8173 & 0.8318 & 0.8592 \\
\hline & Our Approach & Semi-Sup & 0.8041 & 0.8325 & 0.8429 & 0.8416 & 0.8457 & 0.8393 & 0.8364 & 0.8592 \\
\hline
\end{tabular}

TABLE II: Five Class Imbalance Situations of Experiments. $S 1$ is the baseline situation containing evenly distributed classes. $S 2$ to $S 5$ are four situations where data of three classes are reduced to 2,000 while data of the rest classes are increased to 7,000 .

\begin{tabular}{|c|c|c|c|c|c|c|}
\hline & Classes & S1 & S2 & S3 & S4 & S5 \\
\hline \multirow{5}{*}{$\begin{array}{l}\text { Class } \\
\text { Distribution }\end{array}$} & Sitting & 4000 & 2000 & 7000 & 7000 & 2000 \\
\hline & Standing & 4000 & 2000 & 2000 & 7000 & 7000 \\
\hline & Walking & 4000 & 2000 & 2000 & 2000 & 7000 \\
\hline & Ascending Stairs & 4000 & 7000 & 2000 & 2000 & 2000 \\
\hline & Descending Stairs & 4000 & 7000 & 7000 & 2000 & 2000 \\
\hline
\end{tabular}

on HAR task, we filter the "other" class and perform fiveclass classification. We design five class-imbalance situations as shown in Table II. $S 1$ is the basic situation containing evenly distributed classes. $S 2$ to $S 5$ are four situations where data of three classes are reduced to 2,000 while data of the rest classes are increased to 7,000. Note that MHEALTH does not include ascending and descending stairs, so we replace them with cycling and climbing stairs. In addition to three state-ofthe-art methods [16], [11], [10], we also compare our method with two baselines, Over-Sampling and Under-Sampling that perform sampling by randomly selecting or filtering out a certain number of samples. For fairness, we use RAMs as the classifiers for comparison.

Table III shows the performance of these methods on four datasets in five class situations. As F1 score is the most suitable measurement for class imbalanced problems, we use the F1 score in this table. After eliminating the impact of "others", the overall classification performance on MHEALTH and PAMAP2 is boosted. Since $S 1$ enjoys even class distribution, we can observe that the overall performance in $S 1$ is higher than that in other situations. Besides, both the sampling models and subspace generation use RAMs and achieve the same performance as ours in the balanced situation, because the classifiers are regularly trained without any strategy. Another observation is that the results in $S 2$ are relatively low because the two activities, ascending and descending stairs, are hard to be distinguished even though there are plenty of data.

Regarding the methods, our approach outperforms the others in all situations, and the difference between the performance in class balanced situation and class imbalanced situations is not apparent. Among the other compared methods, sampling models and subspace generation have the most similar performance to our model owing to the same classifiers that they use, and subspace generation is more robust on imbalanced data. It is hard to distinguish which sampling strategy is better: under-sampling simply throws out the information while oversampling may repeat some samples many times and lead to overfitting of models. One-class classification is supposed to perform well on imbalanced datasets, but the performance is not that outstanding. Although the method is not affected by the class distribution, the insufficient data of those reduced 
TABLE III: The F1 Scores of All Compared Methods on Five Different Class Situations. The details about $S 1$ to $S 5$ are listed in Table II.

\begin{tabular}{|c|c|c|c|c|c|c|c|c|c|c|c|c|}
\hline & \multicolumn{6}{|c|}{ MHEALTH } & \multicolumn{6}{|c|}{ PAMAP2 } \\
\hline & Over- & Under- & One-Class & Subspace & Ensemble & Our & Over- & Under- & One-Class & Subspace & Ensemble & Our \\
\hline & Sampling & Sampling & [16] & Generation [11] & LSTM [10] & Approach & Sampling & Sampling & [16] & Generation [11] & LSTM [10] & Approach \\
\hline S1 & 0.9921 & 0.9921 & 0.7975 & 0.9921 & 0.9338 & 0.9921 & 0.9287 & 0.9287 & 0.7320 & 0.9287 & 0.8704 & 0.9287 \\
\hline S2 & 0.9425 & 0.9580 & 0.7630 & 0.9627 & 0.9052 & 0.9832 & 0.8818 & 0.8679 & 0.7064 & 0.9024 & 0.8350 & 0.9207 \\
\hline S3 & 0.9672 & 0.9659 & 0.7712 & 0.9715 & 0.9078 & 0.9854 & 0.8858 & 0.8746 & 0.7152 & 0.9134 & 0.8435 & 0.9143 \\
\hline S4 & 0.9608 & 0.9605 & 0.7756 & 0.9874 & 0.9184 & 0.9916 & 0.8903 & 0.8867 & 0.7281 & 0.9157 & 0.8361 & 0.9141 \\
\hline \multirow[t]{4}{*}{ S5 } & 0.9590 & 0.9735 & 0.7673 & 0.9824 & 0.9139 & 0.9834 & 0.8910 & 0.8939 & 0.7176 & 0.9093 & 0.8526 & 0.9223 \\
\hline & \multicolumn{6}{|c|}{ UCI HAR } & \multicolumn{6}{|c|}{ MARS } \\
\hline & Over- & Under- & One-Class & Subspace & Ensemble & Our & Over- & Under- & One-Class & Subspace & Ensemble & Our \\
\hline & Sampling & Sampling & [16] & Generation [11] & LSTM & Approach & Sampling & Sampling & [16] & Generation [11] & LSTM & Approach \\
\hline S1 & 0.7212 & 0.7212 & 0.6255 & 0.7212 & 0.6804 & 0.7212 & 0.8437 & 0.8437 & 0.7383 & 0.8437 & 0.8273 & 0.8437 \\
\hline S2 & 0.6797 & 0.6615 & 0.5619 & 0.7026 & 0.6641 & 0.7013 & 0.8097 & 0.8114 & 0.6983 & 0.8158 & 0.8002 & 0.8312 \\
\hline S3 & 0.6820 & 0.6959 & 0.5844 & 0.7060 & 0.6613 & 0.7191 & 0.8265 & 0.8201 & 0.6943 & 0.8205 & 0.7945 & 0.8349 \\
\hline S4 & 0.6947 & 0.6967 & 0.6086 & 0.7095 & 0.6820 & 0.7099 & 0.8090 & 0.8219 & 0.6986 & 0.8377 & 0.8133 & 0.8314 \\
\hline S5 & 0.6836 & 0.6732 & 0.5927 & 0.7048 & 0.6644 & 0.7159 & 0.8038 & 0.8235 & 0.6925 & 0.8283 & 0.8079 & 0.8564 \\
\hline
\end{tabular}

TABLE IV: Ablation Study. The table presents the accuracy, training time (s) and test time (ms) of the models on 2000 balanced training data and the F1 scores on 5000 imbalanced training data. The imbalanced ratio is as S2. The training time is shown in the parenthesis.

\begin{tabular}{|c|c|c|c|c|c|c|c|c|}
\hline Ablation & Datasets & Balanced & Imbalanced & $\begin{array}{l}\text { Test Time } \\
(\mathrm{ms})\end{array}$ & Datasets & Balanced & Imbalanced & $\begin{array}{l}\text { Test Time } \\
(\mathrm{ms})\end{array}$ \\
\hline RAM & & $0.8327(121.84)$ & $0.8329(182.98)$ & 24.0 & & $0.6863(94.31)$ & $0.6084(124.78)$ & 19.7 \\
\hline Co-Training & & $0.6495(127.42)$ & $0.7208(142.60)$ & 8.9 & & $0.5739(116.94)$ & $0.552(121.28)$ & 7.6 \\
\hline Pattern-Balanced Training & MHEALTH & $0.7462(305.93)$ & $0.9232(621.85)$ & 8.9 & UCI HAR & $0.6533(274.12)$ & $0.6854(554.35)$ & 7.6 \\
\hline Co-Training+RAM & & $0.9123(445.05)$ & $0.7753(674.14)$ & 24.0 & & $0.7503(382.04)$ & $0.5839(574.91)$ & 19.7 \\
\hline Our Approach & & $0.9194(503.91)$ & $0.9832(719.08)$ & 24.0 & & $0.7762(450.71)$ & $0.7013(615.05)$ & 19.7 \\
\hline RAM & & $0.7305(184.25)$ & $0.7359(222.01)$ & 27.8 & & $0.7444(126.83)$ & $0.7148(163.22)$ & 21.5 \\
\hline Co-Training & & $0.6042(212.38)$ & $0.6837(146.11)$ & 10.3 & & $0.6538(118.22)$ & $0.6104(143.27)$ & 8.2 \\
\hline Pattern-Balanced Training & PAMAP2 & $0.6453(471.48)$ & $0.8629(640.47)$ & 10.3 & MARS & $0.737(304.32)$ & $0.7972(606.97)$ & 8.2 \\
\hline Co-Training+RAM & & $0.8085(513.21)$ & $0.7043(683.27)$ & 27.8 & & $0.8233(434.29)$ & $0.6376(644.72)$ & 21.5 \\
\hline Our Approach & & $0.8125(623.83)$ & $0.9027(753.98)$ & 27.8 & & $0.8325(517.84)$ & $0.8312(697.19)$ & 21.5 \\
\hline
\end{tabular}

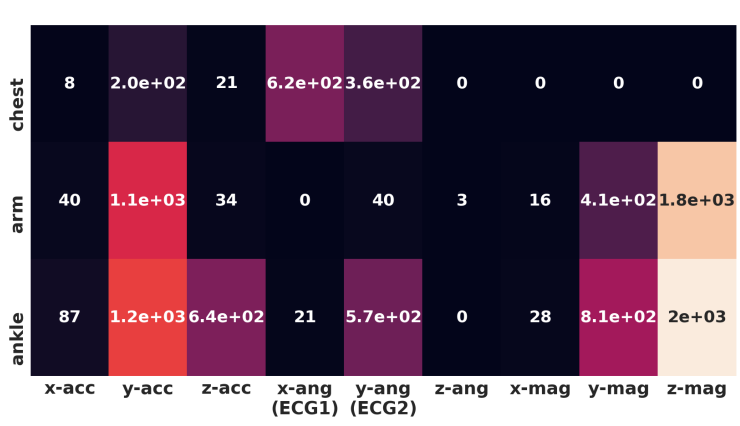

(a) Standing

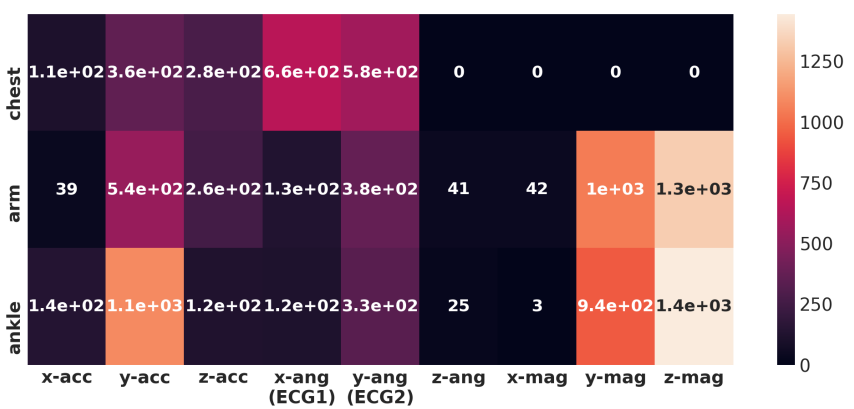

(c) Lying

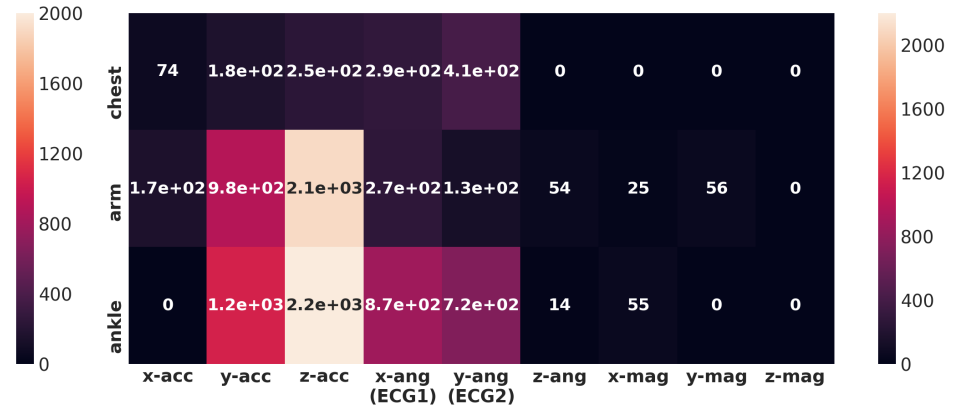

(b) Going Upstairs

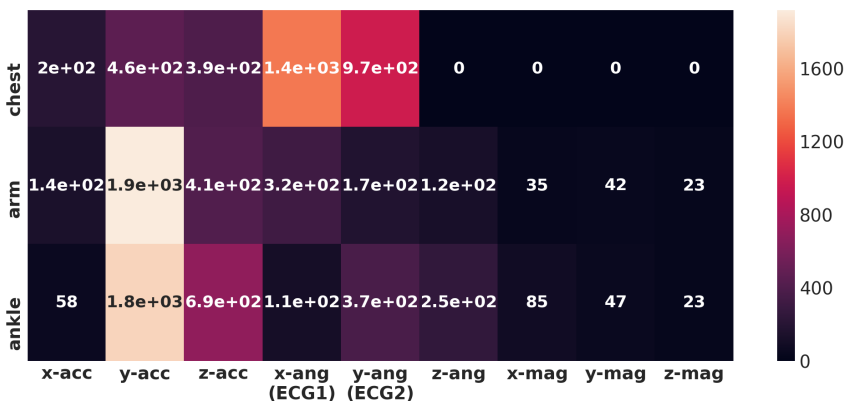

(d) Running

Fig. 4: Visualization of the selected glimpses on MHEALTH. Three rows represent modalities collected from chests, arms and ankles, respectively. Each column denotes one modality. Acc, Ang and Magn denote acceleration, angular velocity and magnetism, respectively. Note that chests only contains 3-axis acceleration and two ECG signals. The values in the grids represent the frequency with which this modality is selected. Lighter colors denote higher frequency.

classes still influence the training process. On the contrary, ensemble LSTM only has satisfactory results in $S 1$, but it 
shows relatively better robustness to imbalanced data.

\section{Ablation Study}

We examine the effectiveness of the proposed components in our method in this section. Table IV presents the accuracy, training time and test time of the ablation models on 2000 balanced data and the F1 scores on 5000 imbalanced training data. The imbalanced ratio is as S2 shown in Table II. To examine the contributions of co-training framework and the pattern balanced training framework without the influence of RAM, we list the performance of these frameworks with regular CNNs. It can be seen that RAM based methods need longer time for test as RAM has more parameters. There is a considerable increase in the training time when RAM is trained with co-training since labeling is conducted in several training rounds. Pattern balanced training further increases the training time as it includes complex data processing. With respect to the performance, it can be observed that RAM in both situations obtains good performance. In the imbalanced situation, pattern balanced training significantly improves the performance than regular co-training since it mitigates the class imbalance. In our experiments, co-training combined with RAM is not that effective on imbalanced data because the imbalance severely influences the F1 scores of classification. In the balanced situation, pattern-balanced training also makes an improvement. The reason of this is that the balanced labeled data may become imbalanced during training rounds, which is avoided by the pattern-balanced training. We can also observe that RAM combined with regular co-training considerably improves the performance with its outstanding voting accuracy. Based on the observations, our method, composing of these components, is comparable with the state-of-the-art approaches.

\section{Visualization of Selected Glimpses}

The attention model extracts salient parts of the input sensory data for recognition, which makes the model explainable. In this section, we present visualized glimpses in recognizing standing, going upstairs, lying and running on MHEALTH. The subjects wear sensors on their chest, dominant arms and ankles, each sensor collecting multiple modalities. The available modalities collected from arms and ankles include 3 -axis acceleration, 3 -axis angular velocity and 3 -axis magnetism. And from chests, the dataset only includes 3 -axis acceleration and two ECG signals.

Fig. 4 shows the glimpse heatmaps of four activities. The glimpses are selected by a well-trained attention model for 10000 times on input data that represent standing, going upstairs, lying and running. The training is on 5000 labeled data and 15000 unlabeled data in a semi-supervised fashion. We observe that when recognizing a specific activity, the model does focus on only a part of modalities. For example, magnetism (orientation) in standing and lying is selected as one of the most active features. And the fact is that it is easy to distinguish between standing and lying with people's orientation. Another example is that the most distinguishing characteristic of going upstairs is "up". Therefore, Z-axis acceleration is specifically selected by agents for going upstairs. Also, identifying running involves acceleration, ECG, and arm swing, which conforms to the experiment evidence as well. The model also selects several other features with lower frequencies, which avoids losing effective information.

\section{CONClusion}

This paper presents an integrated semi-supervised activity recognition system based on multimodal wearable sensor data and addresses a rarely explored problem, i.e., semi-supervised learning for imbalanced human activity recognition (HAR). We first propose a co-training framework that balances the latent patterns of activity data, and then deploy recurrent convolutional attention models as classifiers to exploit unlabeled samples. Comprehensive experiments conducted on four datasets validate the robustness and reliability of the proposed method.

\section{REFERENCES}

[1] D. Anguita, A. Ghio, L. Oneto, X. Parra, and J. L. Reyes-Ortiz, "A public domain dataset for human activity recognition using smartphones." in ESANN, 2013.

[2] A. Bulling, U. Blanke, and B. Schiele, "A tutorial on human activity recognition using body-worn inertial sensors," ACM Computing Surveys (CSUR), vol. 46, no. 3, p. 33, 2014.

[3] H. Guo, L. Chen, L. Peng, and G. Chen, "Wearable sensor based multimodal human activity recognition exploiting the diversity of classifier ensemble," in Proceedings of the 2016 ACM International Joint Conference on Pervasive and Ubiquitous Computing. ACM, 2016, pp. 1112-1123.

[4] J. Yang, M. N. Nguyen, P. P. San, X. Li, and S. Krishnaswamy, "Deep convolutional neural networks on multichannel time series for human activity recognition." in IJCAI Proceedings-International Joint Conference on Artificial Intelligence, 2015, pp. 3995-4001.

[5] L. Yao, F. Nie, Q. Z. Sheng, T. Gu, X. Li, and S. Wang, "Learning from less for better: semi-supervised activity recognition via shared structure discovery," in Ubicomp. ACM, 2016, pp. 13-24.

[6] M. Stikic, K. Van Laerhoven, and B. Schiele, "Exploring semisupervised and active learning for activity recognition," in Wearable computers, 2008. ISWC 2008. IEEE, 2008, pp. 81-88.

[7] A. Blum and T. Mitchell, "Combining labeled and unlabeled data with co-training," in Proceedings of the eleventh annual conference on Computational learning theory. ACM, 1998, pp. 92-100.

[8] D. Guan, W. Yuan, Y.-K. Lee, A. Gavrilov, and S. Lee, "Activity recognition based on semi-supervised learning," in 13th IEEE International Conference on Embedded and Real-Time Computing Systems and Applications (RTCSA 2007). IEEE, 2007, pp. 469-475.

[9] Z.-H. Zhou, D.-C. Zhan, and Q. Yang, "Semi-supervised learning with very few labeled training examples," in AAAI, 2007, pp. 675-680.

[10] Y. Guan and T. Plötz, "Ensembles of deep lstm learners for activity recognition using wearables," Proceedings of the ACM on Interactive, Mobile, Wearable and Ubiquitous Technologies, vol. 1, no. 2, p. 11, 2017.

[11] S. Li, Z. Wang, G. Zhou, and S. Y. M. Lee, "Semi-supervised learning for imbalanced sentiment classification," in IJCAI ProceedingsInternational Joint Conference on Artificial Intelligence, vol. 22, no. 3, 2011, p. 1826.

[12] V. Mnih, N. Heess, A. Graves et al., "Recurrent models of visual attention," in Advances in neural information processing systems, 2014, pp. 2204-2212.

[13] M. Stikic, D. Larlus, and B. Schiele, "Multi-graph based semisupervised learning for activity recognition," in Wearable Computers, 2009. ISWC'09. International Symposium on. IEEE, 2009, pp. 85-92.

[14] N. Y. Hammerla, J. Fisher, P. Andras, L. Rochester, R. Walker, and T. Plötz, "Pd disease state assessment in naturalistic environments using deep learning." in $A A A I, 2015$, pp. 1742-1748.

[15] M. Zeng, T. Yu, X. Wang, L. T. Nguyen, O. J. Mengshoel, and I. Lane, "Semi-supervised convolutional neural networks for human activity recognition," in Big Data. IEEE, 2017, pp. 522-529. 
[16] P. Juszczak and R. P. Duin, "Uncertainty sampling methods for oneclass classifiers," in Proceedings of ICML-03, Workshop on Learning with Imbalanced Data Sets II, 2003, pp. 81-88.

[17] M. Frasca, A. Bertoni, M. Re, and G. Valentini, "A neural network algorithm for semi-supervised node label learning from unbalanced data," Neural Networks, vol. 43, pp. 84-98, 2013.

[18] D. Bahdanau, J. Chorowski, D. Serdyuk, P. Brakel, and Y. Bengio, "Endto-end attention-based large vocabulary speech recognition," in Acoustics, Speech and Signal Processing (ICASSP), 2016 IEEE International Conference on. IEEE, 2016, pp. 4945-4949.

[19] H. Larochelle and G. E. Hinton, "Learning to combine foveal glimpses with a third-order boltzmann machine," in Advances in neural information processing systems, 2010, pp. 1243-1251.

[20] K. Chen, L. Yao, X. Wang, D. Zhang, T. Gu, Z. Yu, and Z. Yang, "Interpretable parallel recurrent neural networks with convolutional attentions for multi-modality activity modeling," in Neural Networks (IJCNN), 2018 International Joint Conference on. IEEE, 2018, pp. $3016-3021$

[21] H. He and E. A. Garcia, "Learning from imbalanced data," IEEE Transactions on knowledge and data engineering, vol. 21, no. 9, pp. 1263-1284, 2009.

[22] U. Maulik and S. Bandyopadhyay, "Performance evaluation of some clustering algorithms and validity indices," IEEE Transactions on Pattern Analysis and Machine Intelligence, vol. 24, no. 12, pp. 1650-1654, 2002.

[23] H. Wang and L. Wang, "Modeling temporal dynamics and spatial configurations of actions using two-stream recurrent neural networks," Conference on Computer Vision and Pa ern Recognition (CVPR), 2017.

[24] N. J. Butko and J. R. Movellan, "I-pomdp: An infomax model of eye movement," in Development and Learning, 2008. ICDL 2008. 7th IEEE International Conference on. IEEE, 2008, pp. 139-144.

[25] R. J. Williams, "Simple statistical gradient-following algorithms for connectionist reinforcement learning," Machine learning, vol. 8, no. 3-4, pp. 229-256, 1992.

[26] V. Radu, C. Tong, S. Bhattacharya, N. D. Lane, C. Mascolo, M. K Marina, and F. Kawsar, "Multimodal deep learning for activity and context recognition," Proceedings of the ACM on Interactive, Mobile, Wearable and Ubiquitous Technologies, vol. 1, no. 4, p. 157, 2018.

[27] Y. Cheng, X. Zhao, R. Cai, Z. Li, K. Huang, and Y. Rui, "Semisupervised multimodal deep learning for rgb-d object recognition." in IJCAI Proceedings-International Joint Conference on Artificial Intelligence, 2016, pp. 3345-3351.

[28] D. Chen, W. Wang, W. Gao, and Z. Zhou, "Tri-net for semi-supervised deep learning," in Proceedings of the Twenty-Seventh International Joint Conference on Artificial Intelligence, IJCAI 2018, July 13-19, 2018, Stockholm, Sweden., 2018, pp. 2014-2020.

[29] O. Banos, R. Garcia, J. A. Holgado-Terriza, M. Damas, H. Pomares, I. Rojas, A. Saez, and C. Villalonga, "mhealthdroid: a novel framework for agile development of mobile health applications," in International Workshop on Ambient Assisted Living. Springer, 2014, pp. 91-98.

[30] A. Reiss and D. Stricker, "Introducing a new benchmarked dataset for activity monitoring," in Wearable Computers (ISWC), 2012 16th International Symposium on. IEEE, 2012, pp. 108-109.

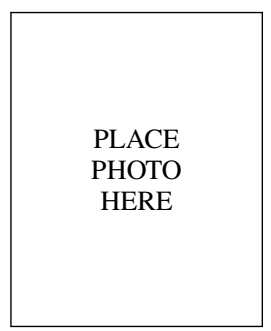

Lina Yao is currently a Senior Lecturer at School of Computer Science and Engineering, The University of New South Wales (UNSW), Australia. Her research interests lie in data mining and machine learning with applications to Internet of Things, information filtering and recommending, human activity recognition and brain computer interface. She is a member of the IEEE and the ACM.

PLACE

PHOTO

HERE
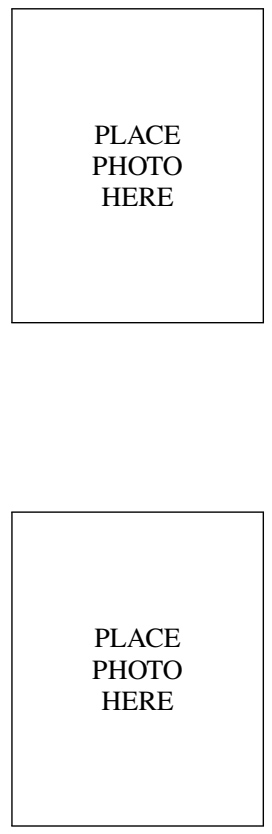

Xiaojun Chang is currently a Lecturer at Monash University. He has received a $\mathrm{PhD}$ degree from the Centre for Quantum Computation and Intelligent Systems (QCIS), University of Technology Sydney, Australia. He is currently a Post Doctoral Associate at the Language Technologies Institute in Carnegie Mellon University. His research interests include machine learning, data mining and computer vision.

PLACE PHOTO

HERE
Xianzhi Wang currently is a Lecturer with School of Software, University Technology of Sydney. His research interests include data management and service-oriented computing. $\mathrm{He}$ is a member of the IEEE and the ACM.
Feiping Nie received the Ph.D. degree in computer science from Tsinghua University, Beijing, China, in 2009. He is currently a Professor with Northwestern Polytechnical University, Xian China. His current research interests include machine learning and its applications, such as pattern recognition, data mining, computer vision, image processing, and information retrieval.
PLACE PHOTO HERE
Kaixuan Chen is a $\mathrm{PhD}$ student at the School of Computer Science and Engineering, University of New South Wales (UNSW) since March, 2017. Her research interests include data mining, machine learning and their applications to Internet of Things, human activity recognition and brain computer interface. School of Computer Science and Engineering, Uni-
versity of New South Wales, Sydney, Australia. His research interest lie in data mining and machine nition, pervasive computing, and brain compute interface. 